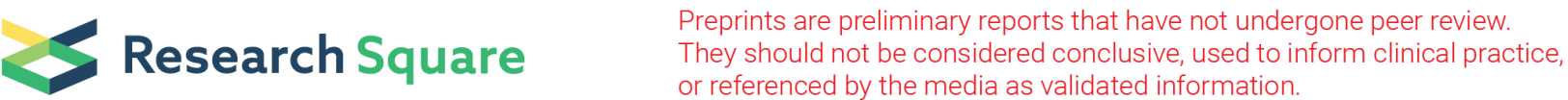

\section{Prognosis of nontuberculous mycobacterial pulmonary disease according to the method of microbiologic diagnosis}

\section{Sooim Sin}

Seoul National University Hospital https://orcid.org/0000-0002-4468-311X

\section{Seungchul Han}

Seoul National University Hospital

\section{Yeon Joo Lee}

Seoul National University Bundang Hospital

\section{Young-jae Cho}

Seoul National University Bundang Hospital

\section{Jong Sun Park}

Seoul National University Bundang Hospital

Ho II Yoon

Seoul National University Bundang Hospital

\section{Choon Taek Lee}

Seoul National University Bundang Hospital

Jae Ho Lee ( $\sim$ jhlee7@snubh.org )

\section{Research}

Keywords: Nontuberculous mycobacterial pulmonary disease, Microbiology, Diagnosis; Bronchoscopy, Sputum, Prognosis

Posted Date: February 11th, 2020

DOl: https://doi.org/10.21203/rs.2.23140/v1

License: (c) (i) This work is licensed under a Creative Commons Attribution 4.0 International License. Read Full License

Version of Record: A version of this preprint was published at Scientific Reports on April 13th, 2021. See the published version at https://doi.org/10.1038/s41598-021-87197-9. 


\section{Abstract}

Background Current microbiologic criteria for nontuberculous pulmonary disease (NTM-PD) require cultures with two separate sputum specimens or one non-sputum specimen. However, data on NTM-PD prognosis following non-sputum culture-based diagnosis are limited. We compared the prognosis of NTM-PD diagnosed using non-sputum and sputum cultures. Methods We analyzed 1020 patients in whom NTM was isolated; they were divided into the sputum NTM isolation $(n=170)$, sputum NTM-PD $(n=688)$, and non-sputum NTM-PD groups $(n=162)$. Initiation of therapy and radiographic aggravation was compared between groups.

Results Overall median observation time was 44.3 months (interquartile range, 28.0-70.2). Fewer nonsputum NTM-PD patients initiated treatment than sputum NTM-PD patients $(p<0.001)$. Moreover, fewer non-sputum NTM-PD patients exhibited radiographic aggravation than sputum NTM-PD patients $(p=0.001)$. Both time to radiographic aggravation and initiation of therapy were significantly shorter in the sputum NTM-PD group (log-rank test; $p=0.011$ and $p<0.001$, respectively). Sputum NTM-PD patients showed higher initiation of therapy $(\mathrm{aHR}, 1.38 ; 95 \% \mathrm{Cl}, 1.02-1.87)$ and risk of radiographic aggravation (aHR, 1.47; $95 \% \mathrm{Cl}, 1.02-2.10)$ than non-sputum NTM-PD patients.

Conclusion Prognosis of NTM-PD patients diagnosed with a non-sputum culture was better than that of NTM-PD patients diagnosed with a sputum culture. The disease course may differ depending on the diagnostic method.

\section{Background}

Recently, the prevalence and mortality of nontuberculous mycobacterial pulmonary disease (NTM-PD) has increased worldwide [1-3]. Therefore, appropriate diagnosis and treatment of NTM-PD is becoming more important. However, pulmonary infection with nontuberculous mycobacteria (NTM) is difficult to diagnose owing to its broad and indolent clinical features. According to the 2007 official American Thoracic Society (ATS) and Infectious Diseases Society of America (IDSA) statements for diagnosis of NTM-PD, microbiologic diagnosis of NTM-PD is typically based on positive results from at least two separate sputum cultures. Alternatively, diagnosis can be made when NTM-PD is suspected on obtaining positive cultures from bronchoscopic specimens or lung tissue when the sputum culture is negative or patients are unable to produce sputum [4]. Although not all patients require treatment, those who meet all the diagnostic criteria, including microbiologic criteria, can be considered for initiation of treatment. However, as NTM is ubiquitous, a single isolation of NTM from expectorated sputum is not considered sufficient for a definitive microbiologic diagnosis $[4,5]$.

Patients with more than one positive sputum culture for NTM exhibit a typical clinical course of pulmonary disease in contrast to patients with only one positive sputum culture [6]. Furthermore, the diagnostic yield of sputum culture compared to that of bronchoscopic specimens for NTM-PD has been investigated in several studies [7-10]. However, no study has yet proven whether patients with NTM 
isolated from bronchoscopic specimens or lung tissue demonstrate disease progression equivalent to that in patients diagnosed using sputum culture. Thus, the microbiologic criteria for NTM-PD is based on low-quality evidence, and it remains to be clarified whether NTM-PD diagnosed following microbiologic criteria other than sputum culture can provide an appropriate prognosis of pulmonary disease. In this study, we compared prognoses of NTM-PD diagnosed using bronchoscopy or lung tissue with prognosis of NTM-PD diagnosed using sputum culture and with a single positive NTM isolation.

\section{Methods}

\section{Study design and population}

This is a retrospective cohort study based on the NTM cohort at Seoul National University Bundang Hospital, which enrolled 1531 patients with NTM isolated from specimens at least once between January 2005 and December 2016. We designated patients with a single NTM isolation from the sputum as the sputum NTM isolation group. Patients who showed negative results on at least two separate sputum specimens and patients who were not able to expectorate sputum underwent additional bronchoscopy. Patients in whom NTM was isolated at least two times from separately expectorated sputum samples were designated as the NTM-PD group diagnosed using sputum culture (sputum NTM-PD group). Finally, patients with positive results from the bronchoscopic specimens (e.g., bronchial washing or bronchoalveolar lavage [BAL]) or lung tissue were designated as the NTM-PD group diagnosed using other methods (non-sputum NTM-PD group).

Patients without baseline radiography or follow-up radiography and those with a follow-up period of less than 1 year were excluded. Additionally, patients diagnosed with active pulmonary tuberculosis within 1 year before or after NTM isolation were excluded (in the sputum NTM-PD group, the time of second positive sputum culture was considered the time of NTM isolation). Patients diagnosed with lung malignancies or interstitial lung disease during the follow-up period were also excluded.

Of the patients in the cohort, 277 were classified into the sputum NTM isolation group, 934 into the sputum NTM-PD group, and 235 into the non-sputum NTM-PD group. Among the eligible patients, 426 were excluded based on the exclusion criteria. Accordingly, the following number of patients were included in the final analysis: 170 in the sputum NTM isolation group, 688 in the sputum NTM-PD group, and 162 in the non-sputum NTM-PD group (Fig. 1).

This study was approved by the Institutional Review Board of the Seoul National University Bundang Hospital (IRB No. B-1812-511-102) and conformed to the tenets of the Declaration of Helsinki.

\section{Data collection}

All results from the patients' acid-fast bacilli (AFB) staining and cultures from January 2005 to December 2016 were reviewed. The time of NTM isolation was defined as time zero. Baseline demographics 
including age and sex, comorbidities, smoking status, and respiratory symptoms within 3 months before or after time zero were collected, and medical records, including prescriptions during the follow-up period, were reviewed in the electronic medical record system. Patient imaging studies, including chest plain radiography and computed tomography (CT) performed during the follow-up period, were thoroughly reviewed for evidence of radiographic aggravation.

\section{Assessment of disease progression}

To compare prognoses between groups, radiographic aggravation and initiation of treatment for NTM-PD were assessed. Radiographic aggravation was assessed independently by a radiologist and a pulmonologist who were blinded to the clinical data. Any discrepancies in decisions were addressed and a final decision was reached by consensus. Presence, severity, and extent of bronchiectasis, cavity, nodules, and consolidation, and the presence of infiltrate are radiographic features associated with NTMPD according to the ATS/IDSA guidelines and previous studies $[4,12,13]$. Chest $X$-ray images were used for evaluation when there was no CT for comparison. We identified patients who began treatment for NTM-PD after time zero, regardless of the treatment period. The proportion of patients with radiographic aggravation and initiation of treatment were compared between groups. The time to radiographic aggravation and treatment initiation were also compared between groups. Moreover, disease progression risk according to the diagnostic methods were estimated after adjustment of variables.

\section{Statistical analyses}

One-way analysis of variance was used for comparison of demographics involving continuous variables, and the chi-squared test and Bonferroni's correction for multiple comparisons were used to compare demographics involving categorical variables. Kaplan-Meier analysis with the log-rank test was performed to compare outcomes between groups. Cox proportional hazard regression analysis was used to calculate adjusted hazard ratios (aHR) and their 95\% confidence intervals (Cl). P-values less than 0.05 were considered statistically significant. Data analysis was conducted using the STATA 13 software (Stata Corp, College Station, Texas).

\section{Results}

\section{Baseline characteristics}

The median observation time was 44.3 months (IQR, 28.0-70.2) for all patients, 38.5 months (IQR, 24.660.4) for the sputum NTM isolation group, 46.4 months (IQR, 30.1-74.2) for the sputum NTM-PD group, and 43.3 months (IQR, 26.0-65.0) for the non-sputum NTM-PD group (calculated after excluding patients treated immediately). 
Baseline characteristics of the subjects are shown in Table 1. There were no significant differences in baseline characteristics, including proportion of sexes, smoking status, history of pulmonary tuberculosis, and comorbidities (including malignancy, diabetes mellitus, chronic renal disease, hypertension, cardiovascular disease), and respiratory symptoms of weight loss between the groups. Patients in the sputum NTM isolation group had a higher body mass index (BMI) than those in other groups. Patients in the non-sputum NTM-PD group were younger, had fewer comorbidities such as chronic obstructive pulmonary disease and asthma, and had fewer complaints of cough or sputum than the other groups. The sputum NTM-PD group included more bronchiectasis patients than the other groups (Table 1). 
Table 1

Baseline characteristics and clinical features of the patients

\begin{tabular}{|c|c|c|c|c|}
\hline Variables & $\begin{array}{l}\text { Sputum NTM isolation } \\
\text { group } \\
(n=170)\end{array}$ & $\begin{array}{l}\text { Non-sputum } \\
\text { NTM-PD } \\
\text { group } \\
(n=162)\end{array}$ & $\begin{array}{l}\text { Sputum } \\
\text { NTM-PD } \\
\text { group } \\
(n=688)\end{array}$ & $\begin{array}{l}\text { P- } \\
\text { value }\end{array}$ \\
\hline Age, years & $63.3 \pm 12.0$ & $59.0 \pm 12.5$ & $64.2 \pm 11.4$ & $<0.001$ \\
\hline Sex, male, n (\%) & $76(44.7)$ & 62 (38.3) & $259(37.6)$ & 0.235 \\
\hline $\mathrm{BMl}, \mathrm{kg} / \mathrm{m} 2$ & $21.7 \pm 3.2$ & $21.5 \pm 2.7$ & $21.1 \pm 2.9$ & 0.020 \\
\hline Smoking, n (\%) & & & & 0.539 \\
\hline Never & $132(77.6)$ & $122(75.3)$ & $545(79.2)$ & \\
\hline Ever smoker & $38(22.4)$ & $40(24.7)$ & $143(20.8)$ & \\
\hline $\begin{array}{l}\text { History of pulmonary } \\
\text { TB }\end{array}$ & $38(22.4)$ & $37(22.8)$ & $202(29.4)$ & 0.074 \\
\hline \multicolumn{5}{|l|}{ Comorbidities, n (\%) } \\
\hline Bronchiectasis & $87(51.2)$ & $79(48.8)$ & $457(66.4)$ & $<0.001$ \\
\hline COPD & $15(8.8)$ & $5(3.1)$ & $64(9.3)$ & 0.033 \\
\hline Asthma & $22(12.9)$ & $8(4.9)$ & $70(10.2)$ & 0.042 \\
\hline Malignancy & $26(15.3)$ & $20(12.3)$ & $101(14.7)$ & 0.702 \\
\hline Diabetes mellitus & $18(10.6)$ & $16(9.9)$ & 75 (10.9) & 0.929 \\
\hline Chronic renal disease & $2(1.2)$ & $5(3.1)$ & $12(1.7)$ & 0.403 \\
\hline Hypertension & $34(20.0)$ & $32(19.7)$ & $156(22.7)$ & 0.597 \\
\hline GERD & $12(7.1)$ & $6(3.7)$ & $33(4.8)$ & 0.341 \\
\hline Cardiovascular & $9(5.3)$ & $6(3.7)$ & $49(7.1)$ & 0.230 \\
\hline \multicolumn{5}{|l|}{ Symptoms, n (\%) } \\
\hline Cough & $69(40.6)$ & $41(25.3)$ & 269 (39.1) & 0.003 \\
\hline Sputum & $68(40.0)$ & $24(14.8)$ & $243(35.3)$ & $<0.001$ \\
\hline
\end{tabular}

Categorical variables are expressed as number (\%); continuous variables are expressed as mean \pm standard deviation

$\mathrm{BMI}=$ body mass index; $\mathrm{COPD}=$ chronic obstructive pulmonary disease; $\mathrm{GERD}$ = gastroesophageal reflux disease, NTM = nontuberculous mycobacteria; NTM-PD = nontuberculous mycobacterial pulmonary disease; TB = tuberculosis. 


\begin{tabular}{|c|c|c|c|c|}
\hline Variables & $\begin{array}{l}\text { Sputum NTM isolation } \\
\text { group } \\
(n=170)\end{array}$ & $\begin{array}{l}\text { Non-sputum } \\
\text { NTM-PD } \\
\text { group } \\
(n=162)\end{array}$ & $\begin{array}{l}\text { Sputum } \\
\text { NTM-PD } \\
\text { group } \\
(n=688)\end{array}$ & $\begin{array}{l}\text { P- } \\
\text { value }\end{array}$ \\
\hline Hemoptysis & $22(12.9)$ & $34(21.0)$ & $146(21.2)$ & 0.048 \\
\hline Dyspnea & 12 (7.1) & $10(6.2)$ & $82(11.9)$ & 0.031 \\
\hline Fever & $14(8.2)$ & $8(4.9)$ & $21(3.1)$ & 0.009 \\
\hline Weight loss & $3(1.8)$ & $3(1.9)$ & $17(2.5)$ & 0.798 \\
\hline \multicolumn{5}{|c|}{$\begin{array}{l}\text { Categorical variables are expressed as number (\%); continuous variables are expressed as mean } \pm \\
\text { standard deviation }\end{array}$} \\
\hline \multicolumn{5}{|c|}{$\begin{array}{l}\mathrm{BMI}=\text { body mass index; } \mathrm{COPD}=\text { chronic obstructive pulmonary disease; GERD = gastroesophageal } \\
\text { reflux disease, NTM = nontuberculous mycobacteria; NTM-PD = nontuberculous mycobacterial } \\
\text { pulmonary disease; } \mathrm{TB}=\text { tuberculosis. }\end{array}$} \\
\hline
\end{tabular}

\section{Characteristics of isolated NTM}

Among the 162 patients in the non-sputum NTM-PD group, 131 (80.9\%) patients had a positive culture from bronchial washing, 16 (9.9\%) patients had a positive culture from BAL, and 15 patients $(9.3 \%)$ had a positive culture from percutaneous needle aspiration of lung tissue. Mycobacterium avium complex was the most frequently isolated strain in all groups, followed by Mycobacterium abscessus (Table 2). The other NTM strains with unspecified names, which are not listed in Table 2, included Mycobacterium chelonae, Mycobacterium gordonae, Mycobacterium phocaicum, Mycobacterium margeritense, Mycobacterium septicum, Mycobacterium terrae, and Mycobacterium lentiflavum. 
Table 2

Methods for isolating NTM, proportion of positive AFB smears, and proportion of NTM isolates

\begin{tabular}{|c|c|c|c|}
\hline & $\begin{array}{l}\text { Sputum NTM isolation group } \\
(n=170)\end{array}$ & $\begin{array}{l}\text { Non-sputum } \\
\text { NTM-PD } \\
\text { group } \\
(n=162)\end{array}$ & $\begin{array}{l}\text { Sputum } \\
\text { NTM-PD group } \\
(n=688)\end{array}$ \\
\hline \multicolumn{4}{|l|}{ Isolated specimen } \\
\hline Sputum, n (\%) & $170(100)$ & - & $688(100)$ \\
\hline Bronchial washing & - & $131(80.9)$ & \\
\hline BAL & - & $16(9.9)$ & \\
\hline PCNA & - & $15(9.3)$ & \\
\hline Positive AFB smear, n (\%) & $4(2.4)$ & $13(8.0)$ & $97(14.1)$ \\
\hline \multicolumn{4}{|l|}{ Isolated organism, n (\%) } \\
\hline Mycobacterium avium & $118(69.4)$ & $86(53.1)$ & $372(54.1)$ \\
\hline Mycobacterium intracellulare & $25(14.7)$ & $33(20.4)$ & $185(26.9)$ \\
\hline Mycobacterium abscessus & $7(4.1)$ & $33(20.4)$ & $71(10.3)$ \\
\hline Mycobacterium kansasii & $2(1.2)$ & $5(3.1)$ & $13(1.9)$ \\
\hline Mycobacterium massiliense & $2(1.2)$ & $0(0)$ & $4(0.6)$ \\
\hline Others & $16(9.4)$ & $5(3.1)$ & $43(6.2)$ \\
\hline
\end{tabular}

\section{Radiographic aggravation}

In the sputum NTM isolation group, 48 (28.2\%) patients showed radiographic aggravation during the follow-up period, compared with 259 (37.6\%) patients in the sputum NTM-PD group and 38 (23.5\%) patients in the non-sputum NTM-PD group. The proportion of patients with radiographic aggravation were significantly different between the groups $(p=0.001)$. Post-hoc analysis revealed that a significant difference between the sputum NTM-PD group and the non-sputum NTM-PD group contributed to the results (Table 3 ). The time to radiographic aggravation was significantly different between the groups (log-rank test; $p=0.011$ ) (Fig. 2). In the multivariable Cox proportional hazard regression, the sputum NTM-PD group remained independently associated with a higher risk of radiologic aggravation (aHR, $1.47 ; 95 \% \mathrm{Cl}, 1.02-2.10)$ relative to the non-sputum NTM-PD group after adjustment for age, sex, smoking history, BMI, presence of comorbidities, and initial respiratory symptoms (Table 4). 
Table 3

Comparison of radiographic aggravation and initiation of treatment for NTM-PD between groups

\begin{tabular}{|c|c|c|c|c|c|c|c|}
\hline & \multirow{2}{*}{$\begin{array}{l}\text { Sputum } \\
\text { NTM } \\
\text { isolation } \\
\text { group } \\
(n=170)\end{array}$} & \multirow{2}{*}{$\begin{array}{l}\text { Non- } \\
\text { sputum } \\
\text { NTM-PD } \\
\text { group } \\
(n=162)\end{array}$} & \multirow{2}{*}{$\begin{array}{l}\text { Sputum } \\
\text { NTM- } \\
\text { PD } \\
\text { group } \\
(\mathrm{n}= \\
688)\end{array}$} & \multicolumn{4}{|l|}{ P-value* } \\
\hline & & & & Overall & $\begin{array}{l}\text { Sputum } \\
\text { NTM } \\
\text { isolation } \\
\text { group vs. } \\
\text { Non- } \\
\text { sputum } \\
\text { NTM-PD } \\
\text { group }\end{array}$ & $\begin{array}{l}\text { Sputum } \\
\text { NTM } \\
\text { isolation } \\
\text { group vs. } \\
\text { Sputum } \\
\text { NTM-PD } \\
\text { group }\end{array}$ & $\begin{array}{l}\text { Non- } \\
\text { sputum } \\
\text { NTM-PD } \\
\text { group vs. } \\
\text { Sputum } \\
\text { NTM-PD } \\
\text { group }\end{array}$ \\
\hline $\begin{array}{l}\text { Radiographic } \\
\text { aggravation, } \\
\text { n(\%) }\end{array}$ & $48(25.8)$ & $38(23.6)$ & $\begin{array}{l}259 \\
(37.6)\end{array}$ & 0.001 & 0.321 & 0.022 & 0.001 \\
\hline $\begin{array}{l}\text { Initiation of } \\
\text { treatment, } n \\
(\%)\end{array}$ & $4(2.4)$ & $57(35.2)$ & $\begin{array}{l}356 \\
(51.7)\end{array}$ & $\begin{array}{l}< \\
0.001\end{array}$ & $<0.001$ & $<0.001$ & $<0.001$ \\
\hline
\end{tabular}

\section{NTM = nontuberculous mycobacteria; NTM-PD = nontuberculous mycobacterial pulmonary disease}


Table 4

Results of multivariable Cox proportional hazards analysis

$\begin{array}{llllll}\text { HR for radiographic } & 95 \% & \text { P- } & \text { HR for initiation } & 95 \% & \begin{array}{l}\text { P- } \\ \text { aggravation }\end{array} \\ \text { Cl } & \text { value } & \text { of treatment } & \text { Cl } & \text { value }\end{array}$

Group

Non-sputum NTM- reference

reference

PD group

Sputum NTM

isolation group

Sputum NTM-PD $\quad 1.47$

group

Age, year

$\mathrm{BMI}, \mathrm{kg} / \mathrm{m}^{2}$

0.89

1.00

0.93

1.02

Ever smoker

Female

Ever smoker

Bronchiectasis

1.57

0.80

0.92

1.77

GERD

Asthma

COPD

nitial symptom

Hemoptysis

0.84

$0.64-$

1.12

$1.12-$

2.8

$0.57-$

1.47

$0.733 \quad 0.94$

$0.733 \quad 0.94$

0.57

0.225

.

0.91

0.001

0.82

1.12

0.64

2.09

0.002

.

0.55
1.15

0.015

0.69

1.21 $0.40-\quad 0.197$

$0.01-<$

$0.15 \quad 0.001$

$1.02-\quad 0.038$

1.87

$0.99-$

0.747

1.01

$0.87-<$

$0.94 \quad 0.001$

$0.62-\quad 0.177$

1.09

$0.82-\quad 0.486$

1.52

$0.51-<$

$0.81 \quad 0.001$

$0.37-\quad 0.006$ 0.85

$0.63-\quad 0.63$

1.40

0.197
Sputum

Cough
0.95

0.79
$0.74-$

1.21

$0.62-$

1.01
$0.240 \quad 1.12$

$0.85-\quad 0.412$

1.48

$0.73-$

1.18

0.981.55

$\mathrm{BMI}=$ body mass index; $\mathrm{COPD}=$ chronic obstructive pulmonary disease; $\mathrm{GERD}=$ gastroesophageal reflux disease, NTM = nontuberculous mycobacteria; NTM-PD = nontuberculous mycobacterial pulmonary disease 


\begin{tabular}{|lllllll|}
\hline & $\begin{array}{l}\text { HR for radiographic } \\
\text { aggravation }\end{array}$ & $\begin{array}{l}95 \% \\
\text { Cl }\end{array}$ & $\begin{array}{l}\text { P- } \\
\text { value }\end{array}$ & $\begin{array}{l}\text { HR for initiation } \\
\text { of treatment }\end{array}$ & $\begin{array}{l}95 \% \\
\text { Cl }\end{array}$ & $\begin{array}{l}\text { P- } \\
\text { value }\end{array}$ \\
\hline Dyspnea & 1.02 & $\begin{array}{l}0.69- \\
1.50\end{array}$ & 0.69 & 1.26 & $0.91-$ & 0.166 \\
\hline \multicolumn{7}{|l|}{$\begin{array}{l}\text { BMI = body mass index; COPD = chronic obstructive pulmonary disease; GERD = gastroesophageal } \\
\text { reflux disease, NTM = nontuberculous mycobacteria; NTM-PD = nontuberculous mycobacterial } \\
\text { pulmonary disease }\end{array}$} \\
\hline
\end{tabular}

\section{Initiation of treatment}

Among all patients, four (2.4\%) in the sputum NTM isolation group, $356(51.7 \%)$ in the sputum NTM-PD group, and 57 (35.2\%) in the non-sputum NTM-PD group began therapy for NTM-PD after time zero. The proportion of patients who initiated treatment was significantly different between the groups $(p<0.001)$, and there were significant differences between all groups (Table 3). The time to initiation of therapy for NTM-PD was significantly different between the groups (log-rank test; $p<0.001$ ) (Fig. 3). In the multivariable Cox proportional hazard regression, the sputum NTM-PD group remained independently associated with a higher risk of initiation of treatment (aHR, $1.38 ; 95 \% \mathrm{Cl}, 1.02-1.87)$ relative to the nonsputum NTM-PD group after adjustment for age, sex, smoking status, BMI, presence of comorbidities, and initial respiratory symptoms (Table 4).

\section{Discussion}

This study investigated whether NTM-PD diagnosed using bronchoscopy or lung tissue biopsy in accordance with the ATS/IDSA guidelines exhibits the typical course of NTM-PD. We compared the prognoses of patients diagnosed with NTM-PD using bronchoscopy or lung tissue biopsy with those of (1) patients not typically diagnosed with NTM-PD due to minimal risk of progression (sputum NTM isolation group) and (2) patients diagnosed with NTM-PD by separately expectorated sputum (sputum NTM-PD group), a typical method for diagnosing NTM-PD. There were significant differences between the non-sputum NTM-PD group and sputum NTM-PD group in the progression of pulmonary disease in terms of radiographic aggravation and initiation of treatment. However, no significant differences were observed between the non-sputum NTM-PD and sputum NTM isolation groups regarding radiographic aggravation.

In this study, the proportion of patients who received treatment in the sputum NTM-PD group was in line with previous studies investigating the natural course of NTM-PD [14, 15]. Furthermore, the proportion of patients who received treatment in the sputum NTM isolation group was similar to that of a study that investigated the natural course of subjects with NTM isolates [15]. In the non-sputum NTM-PD group, the proportion was between that of the sputum NTM-PD group and the sputum NTM isolation group. This suggests that prognosis of NTM-PD diagnosed using bronchoscopy may be better than that of NTM-PD diagnosed using separately expectorated sputum. To some extent, the prognosis of NTM-PD diagnosed using bronchoscopy may be worse than that of the sputum NTM isolation group. Interestingly, in terms of 
radiographic aggravation, the non-sputum NTM-PD group clearly had worse prognosis than that of the sputum NTM-PD group; however, the prognosis was similar to that the sputum NTM isolation group. The decision to initiate treatment for suspected NTM-PD often depends on the guidelines [16]. Considering this, the high proportion of patients who initiated treatment in the non-sputum NTM-PD group may not indicate a more advanced disease compared to that in the sputum NTM isolation group.

In several studies, the usefulness of bronchoscopy in detecting NTM or tuberculosis in respiratory specimens was shown to be superior to that of sputum culture [8, 9, 17-19]. In one study, patients with NTM isolates were classified into an NTM lung disease group and a contamination group. In contrast to the NTM disease group, in the contamination group, NTM was more frequently isolated from bronchoscopic specimens than from sputum [20]. In accordance with these studies, our data also suggest that bronchoscopic culture is likely to be more sensitive for detection of NTM strains than sputum culture, regardless of the bacterial burden. Colonization of the lower respiratory tract by various microorganisms occurs through diverse mechanisms, including impaired immunity in patients with underlying lung disease such as cystic fibrosis bronchiectasis and non-cystic fibrosis bronchiectasis [21, 22]. Previous studies also showed that NTM was isolated in a significant proportion of these patients [23-26]. Therefore, isolation of NTM from bronchial washing or BAL may indicate colonization, especially in patients with underlying lung disease. Furthermore, patients with underlying lung disease frequently have symptoms and radiographic findings that are difficult to distinguish from that of NTMPD. Therefore, such patients are more likely to be misdiagnosed with NTM-PD. In fact, the majority of patients enrolled in this study had a history of pulmonary TB or an underlying lung disease, such as bronchiectasis, which is difficult to differentiate from NTM-PD; these patients could have been diagnosed and treated for NTM-PD. Consequently, some of these patients may not have been treated without an official diagnosis of NTM-PD.

In this study, factors other than the diagnostic method associated with disease progression, including low $\mathrm{BMI}$, presence of bronchiectasis, and gastroesophageal reflux disease (GERD) at the time of diagnosis of NTM-PD, were identified in the multivariable analysis. Low BMI is associated with an increased risk of disease progression in NTM-PD [14, 27-29]. Bronchiectasis is also associated with development of NTM$P D$, and some studies have reported an association with disease progression [25, 30, 31]. However, in this study, bronchiectasis showed a protective effect on the initiation of treatment while simultaneously being a risk factor of radiologic aggravation. This could be explained by similar symptoms and radiologic characteristics in bronchiectasis and NTM-PD, which might have prevented the initiation of treatment. Previously, although several studies suggested that the prevalence of GERD is high in NTM-PD patients, there were no data on its association with disease progression [32,33]. This study is the first to suggest a link between GERD and the progression of NTM-PD, and further research is needed for validation.

There is a lack of data regarding the long-term prognosis of NTM-PD. Moreover, to the best of our knowledge, there is no data evaluating the long-term prognosis of NTM-PD diagnosed using microbiologic methods other than sputum culture. In this study, for the first time, we investigated the prognosis of a large number of patients with NTM- cases diagnosed using bronchoscopy or lung tissue 
biopsy. In addition to simple proportional analysis being one of the strengths of our study, Kaplan-Meier analysis and Cox proportional hazards analysis were also used to account for the time of disease progression and adjustment of other factors.

The retrospective design of our study was one of its major limitations because a considerable number of patients could not be followed up for a longer period of time or evaluated for disease progression. Nevertheless, the median follow-up period was longer in our study than that in other studies. Furthermore, the number of sputum cultures was different for each patient during the follow-up period due to the study design. Another limitation was a lack of standardization in the radiologic evaluation methods and protocols used for microbiologic confirmation. For example, some patients were evaluated using CT scans from diverse vendors, while other patients were evaluated using X-ray images.

\section{Conclusion}

In conclusion, patients diagnosed with NTM-PD using bronchoscopic or lung tissue specimens showed no significant difference in disease progression from patients in whom NTM was isolated only once from sputum. However, patients diagnosed with NTM-PD using bronchoscopic or lung tissue specimens had significantly better prognoses than patients diagnosed with NTM-PD using sputum culture. This suggests that the natural course of patients diagnosed with NTM-PD using methods other than sputum culture may not be comparable to that of patients with typical NTM-PD. Further multicenter prospective studies are needed to investigate the long-term prognosis of NTM-PD according to the method of microbiologic diagnosis. Further research in this aspect will contribute a proper revision of the guidelines and more accurate NTM diagnosis and treatment.

\section{Abbreviations}

AFB

acid-fast bacilli; $\mathrm{BAL}=$ bronchoalveolar lavage; $\mathrm{BMI}=$ body mass index; $\mathrm{COPD}=$ chronic obstructive pulmonary disease $\mathrm{CT}$ = computed tomography; NTM = nontuberculous mycobacteria; NTM-PD = nontuberculous mycobacterial pulmonary disease; MAC = Mycobacterium avium complex;

\section{Declarations}

\section{Funding}

This research received no funding.

\section{Competing interests}

The authors declare no conflict of interest. 


\section{Author Contributions}

J.H.L contributed to the study conception and design, interpretation, review, and revision of the manuscript; S.S contributed to the collection and analysis of data, literature search, and writing and revision of the manuscript; S.H reviewed chest imaging scans; C.T.L and J.S.P contributed to the study design and data collection, Y.J.L, Y.C and H.I.Y contributed to the collection and analysis of data. All authors provided final approval for publication submission and critically revised the manuscript for important intellectual content.

\section{Ethics approval and consent to participate}

This study was approved by the Institutional Review Board of the Seoul National University Bundang Hospital (IRB No. B-1812-511-102) and conformed to the tenets of the Declaration of Helsinki.

\section{Consent for publication}

Not applicable

\section{Availability of data and materials}

The datasets used and/or analysed during the current study are available from the corresponding author on reasonable request.

\section{Acknowledgements}

Not applicable.

\section{References}

1. Prevots DR, Marras TK. Epidemiology of human pulmonary infection with nontuberculous mycobacteria: a review. Clin Chest Med. 2015;36(1):13-34.

2. Morimoto $\mathrm{K}$, Iwai $\mathrm{K}$, Uchimura $\mathrm{K}$, Okumura $\mathrm{M}$, Yoshiyama $\mathrm{T}$, Yoshimori $\mathrm{K}$, et al. A steady increase in nontuberculous mycobacteriosis mortality and estimated prevalence in Japan. Ann Am Thorac Soc. 2014;11(1):1-8. 
3. Henkle E, Hedberg K, Schafer S, Novosad S, Winthrop KL. Population-based Incidence of Pulmonary Nontuberculous Mycobacterial Disease in Oregon 2007 to 2012. Ann Am Thorac Soc. 2015;12(5):642-7.

4. Griffith DE, Aksamit T, Brown-Elliott BA, Catanzaro A, Daley C, Gordin F, et al. An official ATS/IDSA statement: diagnosis, treatment, and prevention of nontuberculous mycobacterial diseases. Am J Respir Crit Care Med. 2007;175(4):367-416.

5. Stout JE, Koh W-J, Yew WW. Update on pulmonary disease due to non-tuberculous mycobacteria. International Journal of Infectious Diseases. 2016;45:123-34.

6. Tsukamura M. Diagnosis of Disease Caused by Mycobacterium avium Complex*. Chest. 1991;99(3):667-9.

7. Sekine A, Saito T, Satoh H, Morishita Y, Tsunoda Y, Tanaka T, et al. Limited value of transbronchial lung biopsy for diagnosing Mycobacterium avium complex lung disease. Clin Respir J. 2017;11(6):1018-23.

8. Jett JR, Cortese DA, Dines DE. The Value of Bronchoscopy in the Diagnosis of Mycobacterial Disease. Chest. 1981;80(5):575-8.

9. Sugihara E, Hirota N, Niizeki T, Tanaka R, Nagafuchi M, Koyanagi T, et al. Usefulness of bronchial lavage for the diagnosis of pulmonary disease caused by Mycobacterium avium-intracellulare complex (MAC) infection. J Infect Chemother. 2003;9(4):328-32.

10. Tanaka E, Amitani R, Niimi A, Suzuki K, Murayama T, Kuze F. Yield of computed tomography and bronchoscopy for the diagnosis of Mycobacterium avium complex pulmonary disease. Am J Respir Crit Care Med. 1997;155(6):2041-6.

11. Haworth CS, Banks J, Capstick T, Fisher AJ, Gorsuch T, Laurenson IF, et al. British Thoracic Society guidelines for the management of non-tuberculous mycobacterial pulmonary disease (NTM-PD). Thorax. 2017;72(Suppl 2):ii1-ii64.

12. Lee G, Lee KS, Moon JW, Koh WJ, Jeong BH, Jeong YJ, et al. Nodular bronchiectatic Mycobacterium avium complex pulmonary disease. Natural course on serial computed tomographic scans. Ann Am Thorac Soc. 2013;10(4):299-306.

13. Lee G, Kim HS, Lee KS, Koh WJ, Jeon K, Jeong BH, et al. Serial CT findings of nodular bronchiectatic Mycobacterium avium complex pulmonary disease with antibiotic treatment. AJR Am J Roentgenol. 2013;201(4):764-72.

14. Hwang JA, Kim S, Jo KW, Shim TS. Natural history of Mycobacterium avium complex lung disease in untreated patients with stable course. Eur Respir J. 2017;49(3).

15. Henkle E, Novosad SA, Shafer S, Hedberg K, Siegel SAR, Ku J, et al. Long-Term Outcomes in a Population-based Cohort with Respiratory Nontuberculous Mycobacteria Isolation. Annals of the American Thoracic Society. 2017;14(7):1120-8.

16. Kimizuka Y, Hoshino Y, Nishimura T, Asami T, Sakakibara Y, Morimoto K, et al. Retrospective evaluation of natural course in mild cases of Mycobacterium avium complex pulmonary disease. PLoS One. 2019;14(4):e0216034. 
17. Gopathi NR, Mandava V, Namballa UR, Makala S. A Comparative Study of Induced Sputum and Bronchial Washings in Diagnosing Sputum Smear Negative Pulmonary Tuberculosis. J Clin Diagn Res. 2016;10(3):0c07-10.

18. Nikbakhsh N, Bayani M, Siadati S. The Value of Bronchoalveolar Lavage in the Diagnosis of Sputum Smear-NegativePulmonary Tuberculosis. Iran J Pathol. 2015;10(1):35-40.

19. Urabe N, Sakamoto S, Sano G, Ito A, Homma S. Characteristics of patients with bronchoscopydiagnosed pulmonary Mycobacterium avium complex infection. $J$ Infect Chemother. 2018;24(10):822-7.

20. Del Giudice G, ladevaia C, Santoro G, Moscariello E, Smeraglia R, Marzo C. Nontuberculous mycobacterial lung disease in patients without HIV infection: a retrospective analysis over 3 years. Clinical Respiratory Journal. 2011;5(4):203-10.

21. Borekci S, Halis AN, Aygun G, Musellim B. Bacterial colonization and associated factors in patients with bronchiectasis. Annals of thoracic medicine. 2016;11(1):55-9.

22. Foweraker JE WD. Microbiology of non-CF bronchiectasis. . European Respiratory Monograph. 2011;52:68-96.

23. Fowler SJ, French J, Screaton NJ, Foweraker J, Condliffe A, Haworth CS, et al. Nontuberculous mycobacteria in bronchiectasis: Prevalence and patient characteristics. Eur Respir J. 2006;28(6):1204-10.

24. Tsuji T, Tanaka E, Yasuda I, Nakatsuka Y, Kaji Y, Yasuda T, et al. Nontuberculous mycobacteria in diffuse panbronchiolitis. Respirology. 2015;20(1):80-6.

25. Maiz L, Giron R, Olveira C, Vendrell M, Nieto R, Martinez-Garcia MA. Prevalence and factors associated with nontuberculous mycobacteria in non-cystic fibrosis bronchiectasis: a multicenter observational study. BMC Infect Dis. 2016;16(1):437.

26. Roux AL, Catherinot E, Ripoll F, Soismier N, Macheras E, Ravilly S, et al. Multicenter study of prevalence of nontuberculous mycobacteria in patients with cystic fibrosis in france. J Clin Microbiol. 2009;47(12):4124-8.

27. Kim SJ, Yoon SH, Choi SM, Lee J, Lee CH, Han SK, et al. Characteristics associated with progression in patients with of nontuberculous mycobacterial lung disease : a prospective cohort study. BMC Pulm Med. 2017;17(1):5.

28. Kitada S, Uenami T, Yoshimura K, Tateishi Y, Miki K, Miki M, et al. Long-term radiographic outcome of nodular bronchiectatic Mycobacterium avium complex pulmonary disease. Int $\mathrm{J}$ Tuberc Lung Dis. 2012;16(5):660-4.

29. Kim SJ, Park J, Lee H, Lee YJ, Park JS, Cho YJ, et al. Risk factors for deterioration of nodular bronchiectatic Mycobacterium avium complex lung disease. Int J Tuberc Lung Dis. 2014;18(6):730-6.

30. Kuroishi S, Nakamura Y, Hayakawa H, Shirai M, Nakano Y, Yasuda K, et al. Mycobacterium avium complex disease: prognostic implication of high-resolution computed tomography findings. Eur Respir J. 2008;32(1):147-52. 
31. Mirsaeidi M, Hadid W, Ericsoussi B, Rodgers D, Sadikot RT. Non-tuberculous mycobacterial disease is common in patients with non-cystic fibrosis bronchiectasis. Int J Infect Dis. 2013;17(11):e1000-4.

32. Sharma P, Singh D, Sharma K, Verma S, Mahajan S, Kanga A. Are We Neglecting Nontuberculous Mycobacteria Just as Laboratory Contaminants? Time to Reevaluate Things. J Pathog. 2018;2018:8907629.

33. Koh W-J, Lee JH, Kwon YS, Lee KS, Suh GY, Chung MP, et al. Prevalence of Gastroesophageal Reflux Disease in Patients With Nontuberculous Mycobacterial Lung Disease. Chest. 2007;131(6):1825-30.

\section{Figures}

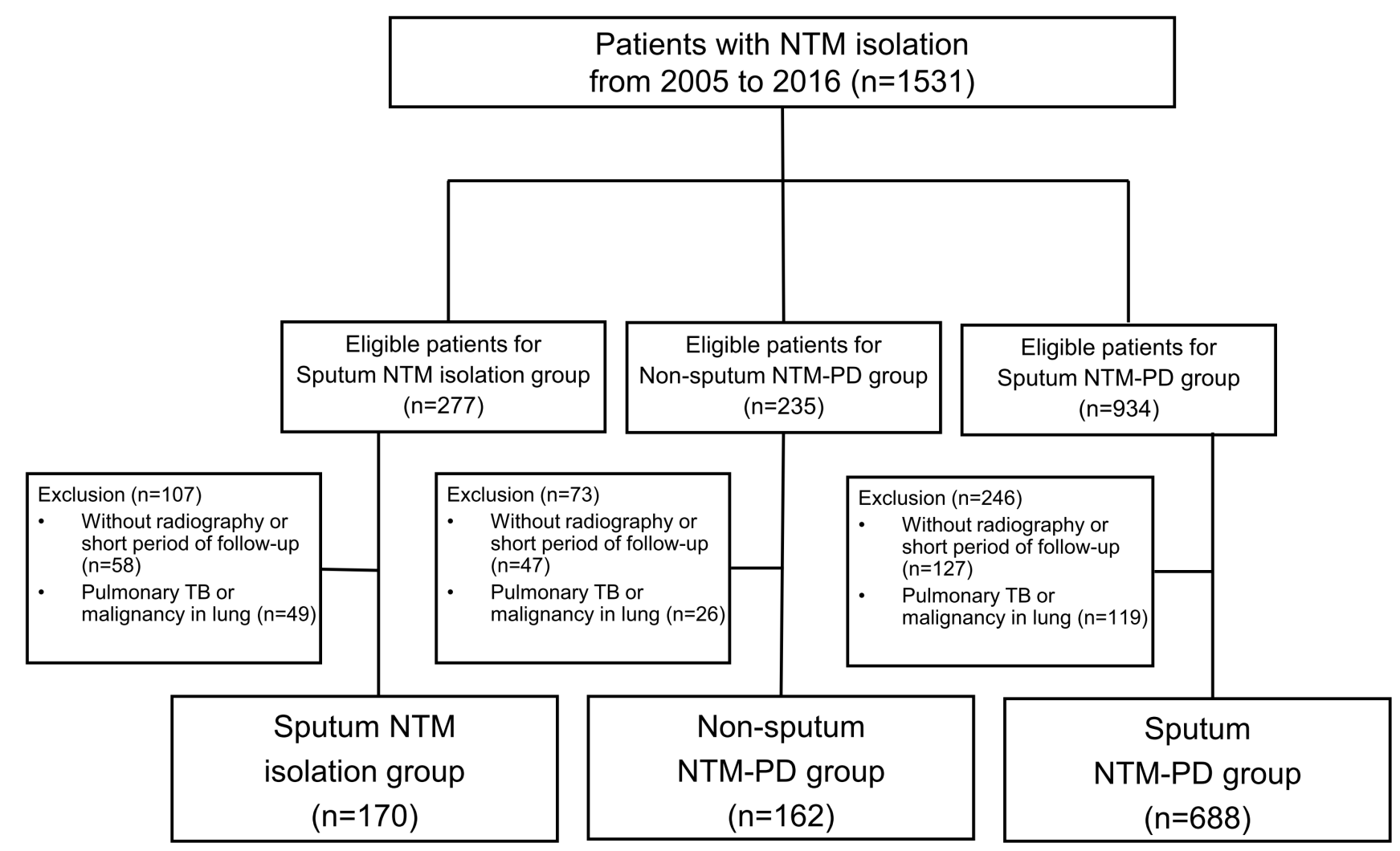

\section{Figure 1}

Flow diagram of the study population enrolled from NTM cohort at Seoul National University Bundang Hospital, from January 2005 to December 2016. NTM = nontuberculous mycobacterium; NTM-PD = nontuberculous mycobacterial pulmonary disease; TB = tuberculosis. Patients with a single NTM isolation from sputum were designated as the sputum NTM isolation group, patients with positive results from bronchoscopic (e.g., bronchial washing or bronchoalveolar lavage [BAL]) or lung tissue specimens were designated as the NTM-PD group diagnosed using other methods (non-sputum NTM-PD group) and 
patients with NTM isolation at least two times from separately expectorated sputum samples were designated as the NTM-PD group diagnosed using sputum culture (sputum NTM-PD group).
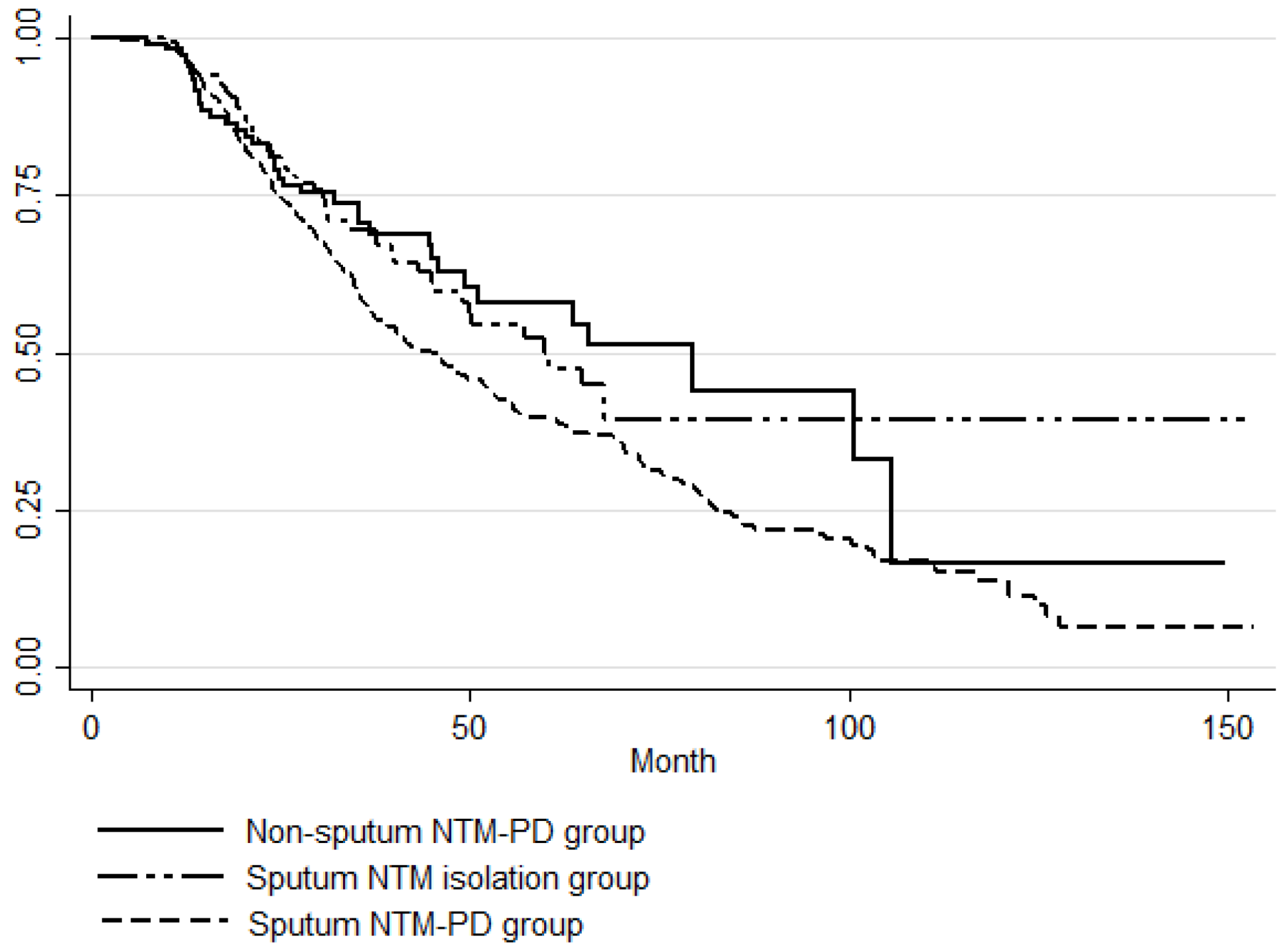

Figure 2

Comparison of time to radiographic aggravation for nontuberculous mycobacterial pulmonary disease (NTM-PD). The duration from the time of nontuberculous mycobacterium (NTM) isolation to the time of radiographic aggravation was significantly different between the groups (Log rank $P=0.027$ ). Patients with a single NTM isolation from sputum were designated as the sputum NTM isolation group, patients with positive results from bronchoscopic (e.g., bronchial washing or bronchoalveolar lavage [BAL]) or lung tissue specimens were designated as the NTM-PD group diagnosed using other methods (nonsputum NTM-PD group), and patients with NTM isolation at least two times from separately expectorated sputum samples were designated as the NTM-PD group diagnosed using sputum culture (sputum NTMPD group). 

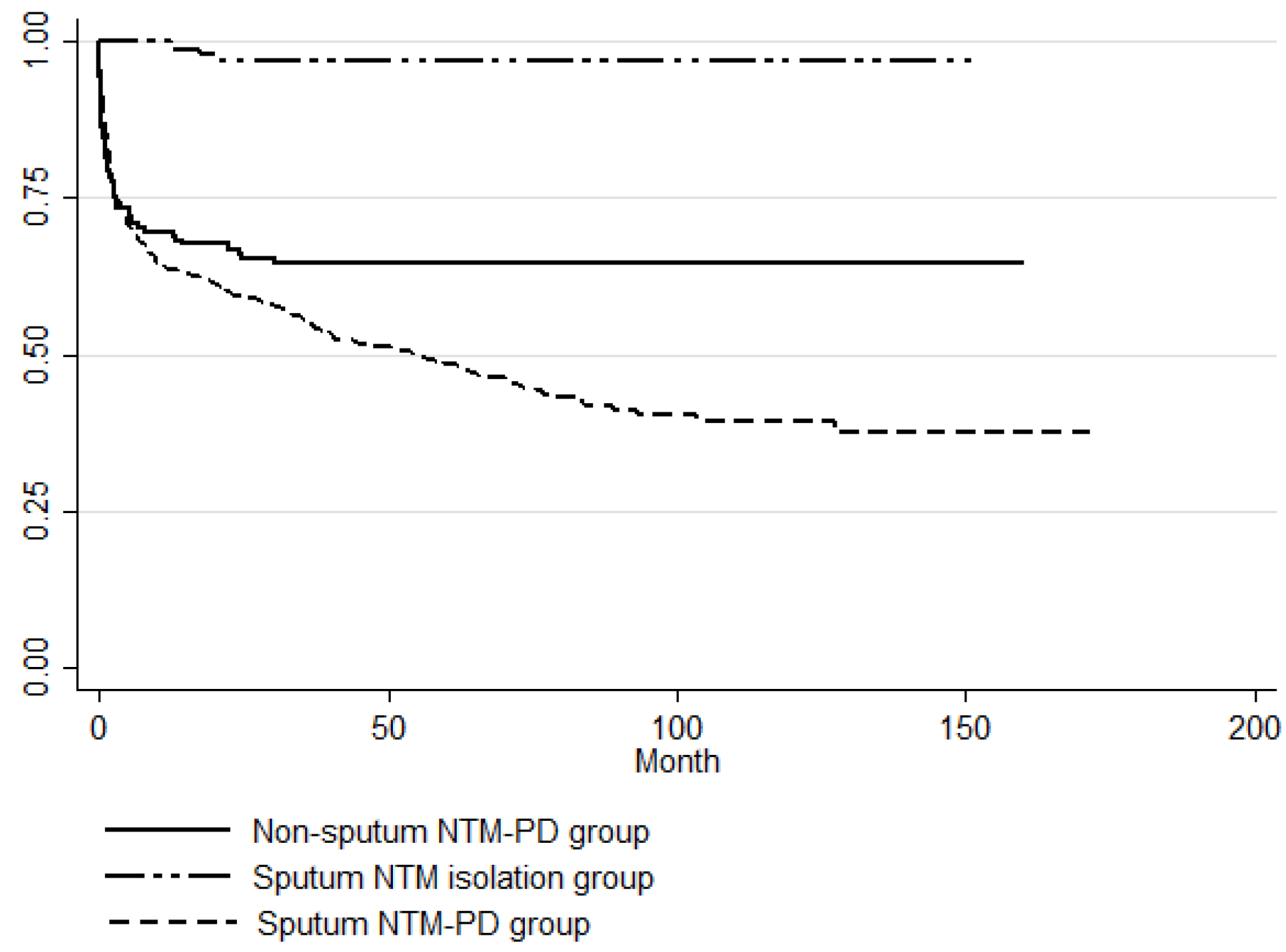

Figure 3

Comparison of time to initiation of treatment for nontuberculous mycobacterial pulmonary disease (NTM-PD). The duration from the time of nontuberculous mycobacterium (NTM) isolation to the time of initiation of NTM-PD treatment was significantly different between the groups (Log rank $P<0.001$ ). Patients with a single NTM isolation from sputum were designated as the sputum NTM isolation group, patients with positive results from bronchoscopic (e.g., bronchial washing or bronchoalveolar lavage [BAL]) or lung tissue specimens were designated as the NTM-PD group diagnosed using other methods (non-sputum NTM-PD group), and patients with NTM isolation at least two times from separately expectorated sputum samples were designated as the NTM-PD group diagnosed using sputum culture (sputum NTM-PD group). 